Central European Journal of Energetic Materials, 2016, 13(2), 301-320

ISSN 1733-7178

e-ISSN 2353-1843

\title{
Synthesis and Characterization of Four Energetic Transition Metal Complexes of 3,4-Diamino-1,2,4-triazole
}

\author{
Xin YIN, Xin JIN, Cai-Xia XU, Piao HE, \\ Kun WANG, Jian-Guo ZHANG*
}

State Key Laboratory of Explosion Science and Technology, Beijing Institute of Technology, Beijing 100081, P. R. China *E-mail: zjgbit@bit.edu.cn

\begin{abstract}
Four complexes with the formula of $\left[\mathrm{M}_{5}(\mathrm{DATr})_{12 \sim 14}\left(\mathrm{H}_{2} \mathrm{O}\right)_{6}\right]\left(\mathrm{ClO}_{4}\right)_{10}$ (M = Mn (1), Co (2), Ni (3), Zn (4)) about their synthesis, structures and some energetic properties (such as sensitivities tests) have been described, where the DATr here denotes 3,4-diamino-1,2,4-triazole. These four compounds are all light metallic complexes with good thermal stability. The structures of 2-4 were determined by single-crystal X-ray diffraction, and the crystal structures mainly consist of penta-nuclear units. All the structures have a common interesting property in which DATr plays a role of bi-dentate ligand. Besides, it is observed from the crystal structure of $\mathbf{4}$ that DATr can be also act as a mono-dentate ligand. Thermodynamic studies of their decomposition properties and kinetic parameters show that the four complexes have high thermal stabilities. Furthermore, tests of their impact and friction sensitivities show that complexes (2) and (3) can be potential candidates as primary explosives to replace toxic lead azide.
\end{abstract}

Keywords: 3,4-diamino-1,2,4-triazole, theoretic study, crystal structure, thermal stability, sensitivity

\section{Introduction}

It is of key importance for new energetic materials (EMs) that they have both fine performance and high security [1]. Recently, research has focused on EMs with the properties of light metallic, mechanical and thermal-dynamic stabilities, replacing environmentally harmful and unstable explosives like $\mathrm{PbN}_{3}$. Compounds of $4^{\text {th }}$ periodic transition metals can meet the requirements 
above. So these compounds have attracted more and more research funding to study this kind of novel EMs [2], especially concentrating on complexes with nitrogen-rich heterocyclic ligands [3], because these ligands have the characters of both high nitrogen content and positive heats of formation.

1,2,4-triazole and its derivates are a kind of nitrogen-rich heterocyclic ligand with fascinating explosive properties [4], due to their diverse coordination modes capable of creating various energetic complexes, such as metal-organic framework compounds (MOF) [5].

Currently, many ligands of 1,2,4-triazole modified with amino group(s) have been studied, such as 4-amino-1,2,4-triazole, 3-hydrazino-4-amino-1,2,4triazole and 3,5-diamino-1,2,4-triazole (Figure 1). These ligands show quite novel structural appearances. For example, the ligand of 4-amino-1,2,4-triazole can be not only a mono-dentate ligand (through N1 atom of triazole) [4d, 4e, 6] but also a bi-dentate ligand (through both $\mathrm{N} 1$ and $\mathrm{N} 2$ atoms of triazole) [4c]. As for 3-hydrazino-4-amino-1,2,4-triazole and 3,5-diamino-1,2,4-triazole, which play roles as tri-dentate [7], bi-dentate ligands [4a, 4f, 8] in the complexes, respectively.
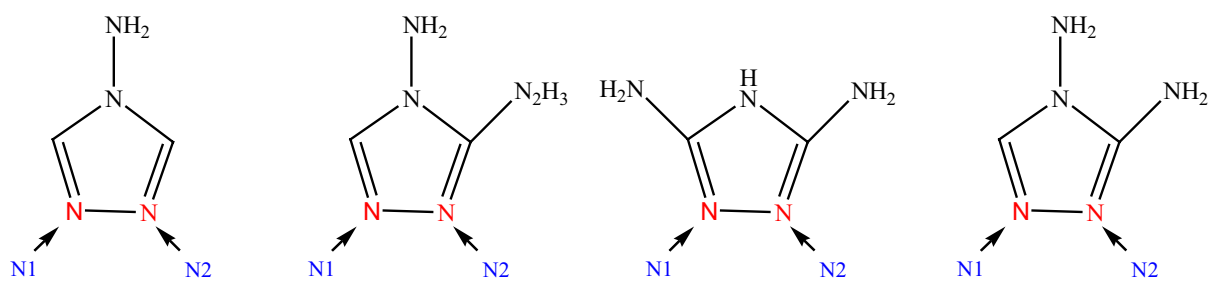

Figure 1. Some common 1,2,4-triazole derivatives with amino groups.

3,4-diamino-1,2,4-triazole (DATr) is a kind of 1,2,4-triazole derivative including two amino groups. Depending on different coordination sites on the triazole ring, DATr can be a mono/bi-dentate ligand. What's more, because of the $\pi$-system and the $-\mathrm{NH}_{2}$ groups, DATr is able to form wide-ranging hydrogen bonds which will contribute to increasing the stability and safety of complexes. However, complexes with DATr as ligands have seldom been reported [9]. We have previously made some contribution to this research.

In our previous work, some energetic complexes based on DATr (as bi-dentate ligands) were synthesized through the reaction of DATr and the corresponding metal nitrates [10]. In order to continue to study its bi-dentate and energetic characters, we presently report four transition metallic complexes of $\left[\mathrm{M}_{5}(\mathrm{DATr})_{12 \sim 14}\left(\mathrm{H}_{2} \mathrm{O}\right)_{6}\right]\left(\mathrm{ClO}_{4}\right)_{10}(\mathrm{M}=\mathrm{Mn}(\mathbf{1}), \mathrm{Co}(\mathbf{2}), \mathrm{Ni}(\mathbf{3}), \mathrm{Zn}(\mathbf{4}))$ including their preparation, crystal structures, thermal decomposition and energetic properties. 


\section{Experimental Section}

\subsection{Materials and general methods}

Caution: The four complexes are potential energetic materials. So we take some protective measures including face shields, leather coat, safety glasses and ear plugs.

All the reagents and starting materials were of analytically pure grades from commercial sources. The 3,4-diamino-1,2,4-triazole (DATr) ligand was prepared according to a published method [11].

Elemental analysis was performed with a Flash EA 1112 full automatic trace element analyzer. The FT-IR spectra were recorded with a Bruker Equinox 55 infrared spectrometer ( $\mathrm{KBr}$ pellets) in the range of $4000-400 \mathrm{~cm}^{-1}$ with a resolution of $4 \mathrm{~cm}^{-1}$. DSC measurements were carried out with a Pyris-1 differential scanning calorimeter in a dry nitrogen gas atmosphere with a flow rate of $20 \mathrm{~mL} \cdot \mathrm{min}^{-1}$.

\subsection{Syntheses}

$30 \mathrm{mmol} \mathrm{LiOH}$ was added to a solution of DATr $\cdot \mathrm{HCl}(30 \mathrm{mmol})$ in water $(30 \mathrm{~mL})$ so as to adjust the acid environment of the DATr solution. Then the solution of $\mathrm{M}\left(\mathrm{ClO}_{4}\right)_{2}(\mathrm{M}=\mathrm{Mn}, \mathrm{Co}, \mathrm{Ni}$ and $\mathrm{Zn})(10 \mathrm{mmol})$ in water $(10 \mathrm{~mL})$ was added slowly to the mixed solution and was vigorously stirred for $0.5 \mathrm{~h}$ at a temperature of $60{ }^{\circ} \mathrm{C}$. Finally the suspension was cooled. The precipitate was filtered off and dried in vacuum.

$\left[\mathrm{Mn}_{5}(\mathrm{DATr})_{12}\left(\mathrm{H}_{2} \mathrm{O}\right)_{6}\right]\left(\mathrm{ClO}_{4}\right)_{10}(\mathbf{1}):$ Mn complex's crystals in the mother liquor were not obtained. Yield: $68.42 \%(3.51 \mathrm{~g})$. Elemental analysis calcd (\%) for $\mathrm{C}_{24} \mathrm{H}_{72} \mathrm{~N}_{60} \mathrm{O}_{46} \mathrm{Cl}_{10} \mathrm{Mn}_{5}$ : C 11.23, H 2.83, N 32.75; found: C 11.57, H 3.96, N 32.39; IR (KBr, cm $\left.{ }^{-1}\right): \mathrm{v}=3474(\mathrm{~s}), 3373(\mathrm{~s}), 3148(\mathrm{~m}), 1642(\mathrm{~s}), 1337(\mathrm{w}), 1087(\mathrm{~s})$, 842(w), 679(w), 627(s), 498(w);

$\left[\mathrm{Co}_{5}(\mathrm{DATr})_{12}\left(\mathrm{H}_{2} \mathrm{O}\right)_{6}\right]\left(\mathrm{ClO}_{4}\right)_{10}(\mathbf{2})$ : After a few days, the red mother liquor started to crystallize. Yield: $84.7 \%(4.38 \mathrm{~g})$. Elemental analysis calcd (\%) for $\mathrm{C}_{24} \mathrm{H}_{72} \mathrm{~N}_{60} \mathrm{O}_{46} \mathrm{Cl}_{10} \mathrm{Co}_{5}$ : C 11.15, H 2.81, N 32.49; found: C 11.17, H 2.89, N 32.43; IR (KBr, $\left.\mathrm{cm}^{-1}\right)$ : v = 3483(s), 3362(s), 3151(w), 1653(s), 1456(w), 1085(s), 839(w), 679(w), 627(s), 511(w);

$\left[\mathbf{N i}_{5}(\mathrm{DATr})_{12}\left(\mathrm{H}_{2} \mathrm{O}\right)_{6}\right]\left(\mathrm{ClO}_{4}\right)_{10}(3)$ : A couple of days later, the blue crystals of nickel complex were obtained from the mother liquor. Yield: 59.01\% (3.05 g). Elemental analysis calcd (\%) for $\mathrm{C}_{24} \mathrm{H}_{72} \mathrm{~N}_{60} \mathrm{O}_{46} \mathrm{Cl}_{10} \mathrm{Ni}_{5}$ : C 11.15, H 2.81, N 32.51; found: C 11.11, H 2.86, N 32.56; IR $\left(\mathrm{KBr}, \mathrm{cm}^{-1}\right): \mathrm{v}=3474(\mathrm{~s}), 3363(\mathrm{~s})$, 3142(m), 1656(s), 1463(w), 1347(w), 1090(s), 854(w), 728(w), 626(s), 518(w);

$\left[\mathrm{Zn}_{5}(\mathrm{DATr})_{14}\left(\mathrm{H}_{2} \mathrm{O}\right)_{2}\right]\left(\mathrm{ClO}_{4}\right)_{10} \cdot 2 \mathrm{H}_{2} \mathrm{O}$ (4): Colorless crystals suitable for $\mathrm{X}$-ray analysis from the mother liquor were obtained after several days. Yield: 
57.91\% (3.22 g). Elemental analysis calcd (\%) for $\mathrm{C}_{28} \mathrm{H}_{78} \mathrm{~N}_{70} \mathrm{O}_{44} \mathrm{Cl}_{10} \mathrm{Zn}_{5}$ : C 12.09, H 2.83, N 35.26; found: C 12.10, H 2.87, N 35.24; IR $\left(\mathrm{KBr}, \mathrm{cm}^{-1}\right): \mathrm{v}=$ 3469(m), 3368(s), 3140(s), 1657(s), 1344(w), 1216(w), 1082(s), 862(m), 727(m), 627(s), 507(w);

$\mathrm{X}$-ray data collection and structure refinement: The data collection was performed with a Rigaku AFC-10/Saturn 724+CCD detector diffractometer with graphite mono-chromated Mo- $K \alpha$ radiation $(\lambda=0.71073 \AA)$ at 293 (2) K with multi-scan modes. The structures were solved by direct methods using SHELXS-97 (Sheldrick, 1990) [12] and refined by full-matrix least-squares methods on $F 2$ with SHELXL-97 program [13]. All non-hydrogen atoms were obtained from the difference Fourier map and refined anisotropically. The hydrogen atoms were generated geometrically and treated by a constrained refinement. The CIF files were checked using the checkCIF website.

Crystallographic data (excluding structure factors) for the structures in this paper have been deposited with the Cambridge Crystallographic Data Centre, CCDC, 12 Union Road, Cambridge CB21EZ, UK. Copies of the data can be obtained free of charge on quoting the depository number CCDC-1004035 for 2, CCDC-1004036 for 3 and CCDC-1004037 for 4 (Fax: +44-1223-336-033; E-mail: deposit@ccdc.cam.ac.uk, http://www.ccdc.cam.ac.uk)

\section{Results and Discussion}

\subsection{Theoretical research}

In order to study the electronic structures and energetic properties of DATr, all calculations were performed using the Gaussian 03 program [14]. The theoretical calculations of DATr were studied using hybrid DFT theory of B3LYP with the basis of 6-311+G**. The optimized structure of DATr is shown in Figure 2.

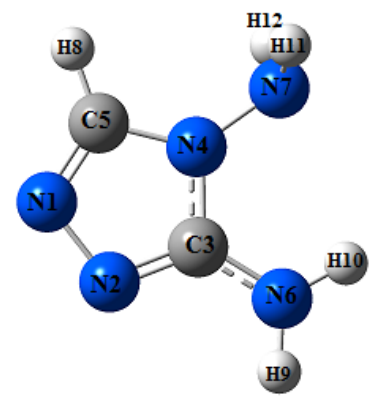

Figure 2. The optimal geometric structure of 3,4-diamino-1,2,4-triazole. 
Based on the optimized structure of DATr, we applied the Multiwfn program to analyse its electrostatic potential surface to further understand its potential coordination sites [15]. This is depicted in Figure 3. Red points and blue points denote maximal values and minimal values of electrostatic potential, respectively.

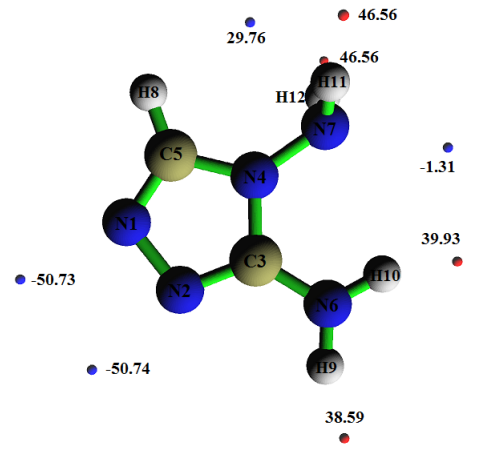

Figure 3. The extreme value distributions of electrostatic potential of 3,4-diamino-1,2,4-triazole.

It can be seen from Figure 3 that there are four maximal value points and four minimal value points due to the electrostatic interactions. Because of the higher electro-negativity of $\mathrm{N}$ atoms compared to $\mathrm{H}$ atoms, positive electrostatic potentials are distributed near $\mathrm{H}$ atoms while negative electrostatic distributions exist around $\mathrm{N}$ atoms. Note that the values of electrostatic potential around $\mathrm{N} 1$ and $\mathrm{N} 2$ atoms of the triazole ring were minimum $\left(-50.73 \mathrm{kcal} \cdot \mathrm{mol}^{-1}\right.$ and $-50.74 \mathrm{kcal} \cdot \mathrm{mol}^{-1}$, respectively), suggesting that $\mathrm{N} 1$ and $\mathrm{N} 2$ atoms in the DATr molecule are likely to coordinate with metal atoms. Therefore, it can be concluded that the two nitrogen atoms ( $\mathrm{N} 1$ and $\mathrm{N} 2)$ of the triazole ring are potential coordination sites, which is consistent with the coordination modes in the crystal structures of complexes $\mathbf{2 - 4}$.

\subsection{Crystal structures}

The single crystals of complexes 2-4 were obtained and determined by singlecrystal X-ray diffraction. Crystallographic data is described in Table 1, and selected bond lengths and angles are listed in Table 2 and Table 3, respectively. 
Table 1. Crystallographic data and structure refinement for complex 2,3 and 4

\begin{tabular}{|c|c|c|c|}
\hline Complex & 2 & \begin{tabular}{|l|}
3 \\
\end{tabular} & 4 \\
\hline Formula & $\mathrm{C} 24 \mathrm{H} 72 \mathrm{~N} 60 \mathrm{O} 46 \mathrm{Cl} 10 \mathrm{Co} 5$ & C24H72N60O46Cl10Ni5 & $\mathrm{C} 28 \mathrm{H} 78 \mathrm{~N} 70 \mathrm{O} 44 \mathrm{Cl10Zn5}$ \\
\hline $\operatorname{Mr}\left[\mathrm{g} \cdot \mathrm{mol}^{-1}\right]$ & 2586.57 & 2585.47 & 2780.95 \\
\hline Crystal system & rhombohedral & trigonal & triclinic \\
\hline Space group & $\mathrm{R}-3$ & R-3 & $\mathrm{P}-1$ \\
\hline$a[\AA]$ & $18.1420(6)$ & $17.9987(11)$ & $10.7655(7)$ \\
\hline$b[\AA]$ & $18.1420(6)$ & $17.9987(11)$ & $11.6784(10)$ \\
\hline$c[\AA]$ & $26.3971(15)$ & $26.148(2)$ & $19.8876(14)$ \\
\hline$\alpha\left[^{\circ}\right]$ & 90 & 90 & $84.559(6)$ \\
\hline$\beta\left[^{\circ}\right]$ & 90 & 90 & $76.593(6)$ \\
\hline$\gamma\left[^{\circ}\right]$ & 120 & 120 & $73.303(7)$ \\
\hline$v\left[\AA^{3}\right]$ & $7524.1(6)$ & $7336.0(9)$ & $2328.6(3)$ \\
\hline $\mathrm{Z}$ & 3 & 3 & 1 \\
\hline$\rho\left[\mathrm{g} \cdot \mathrm{cm}^{-3}\right]$ & 1.713 & 1.756 & 1.983 \\
\hline $\mathrm{M}\left[\mathrm{mm}^{-1}\right]$ & 9.807 & 4.612 & 1.686 \\
\hline $\mathrm{F}(000)$ & 3927 & 3942 & 1408 \\
\hline Crystal size $\left[\mathrm{mm}^{3}\right]$ & $0.15 \times 0.13 \times 0.13$ & $0.14 \times 0.13 \times 0.10$ & $0.11 \times 0.09 \times 0.08$ \\
\hline $\mathrm{T}[\mathrm{K}]$ & $293(2)$ & $293(2)$ & $293(2)$ \\
\hline$\theta$ range $\left[{ }^{\circ}\right]$ & 3.27 to 66.05 & 3.30 to 65.99 & 2.59 to 25.02 \\
\hline Dataset $[\mathrm{h}, \mathrm{k}, \mathrm{l}]$ & $\begin{array}{l}-20 \leq \mathrm{h} \leq 11 \\
-19 \leq \mathrm{k} \leq 15 \\
-28 \leq \mathrm{l} \leq 31\end{array}$ & $\begin{array}{l}-21 \leq \mathrm{h} \leq 8 \\
-9 \leq \mathrm{k} \leq 21 \\
-30 \leq \mathrm{l} \leq 22\end{array}$ & $\begin{array}{l}-12 \leq \mathrm{h} \leq 12 \\
-12 \leq \mathrm{k} \leq 13 \\
-23 \leq 1 \leq 23\end{array}$ \\
\hline $\mathrm{R} 1, \mathrm{wR}_{2}[\mathrm{I}>2 \sigma(\mathrm{I})]$ & $0.1009,0.2317$ & $0.1296,0.2803$ & $0.0808,0.1564$ \\
\hline $\mathrm{R} 1, \mathrm{wR}_{2}$ (all data) & $0.1741,0.2735$ & $0.2582,0.3377$ & $0.1551,0.2142$ \\
\hline Goodness of fit & 1.045 & 1.081 & 1.053 \\
\hline Max (trans) & 0.3621 & 0.6556 & 0.8769 \\
\hline Min (trans) & 0.3209 & 0.5645 & 0.8363 \\
\hline Correction type & multi-scan & multi-scan & multi-scan \\
\hline
\end{tabular}

All three crystal structures mainly consist of penta-nuclear units (shown in Figure 4 for complex 2, Figure 6 for complex 3 and Figure 8 for complex 4, respectively). The penta-nuclear units of the three complexes are centrosymmetric with the inversion center being located at the middle $\mathrm{M}(1)$ atom $(\mathrm{M}=\mathrm{Co}, \mathrm{Ni}, \mathrm{Zn})$. DATr ligands in the three crystal structures mainly bridge a pair of adjacent metal atoms via the $\mathrm{N} 1$ and $\mathrm{N} 2$ atoms of triazole rings, and the ligands of the three complexes behave similarly. 


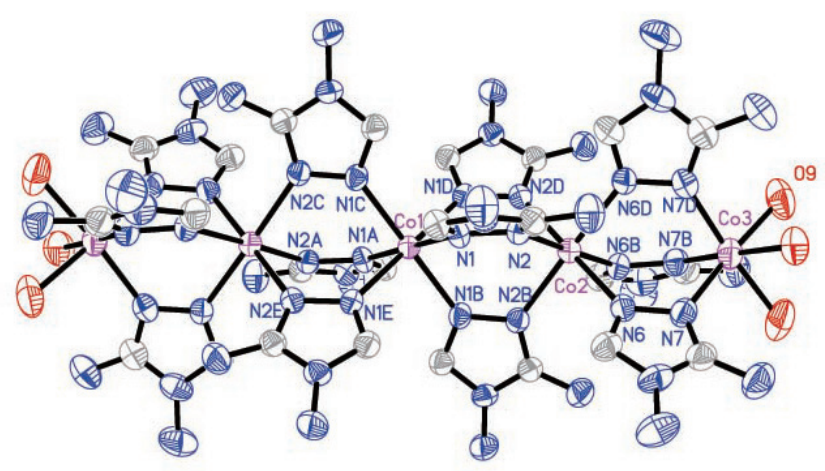

Figure 4. The pentra-nuclear structure of complex 2, thermal ellipsoids drawn at $50 \%$ probability level.

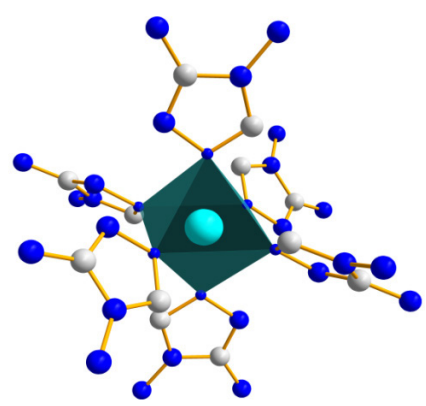

(a) $\mathrm{Co} 1$ and $\mathrm{Co} 2$

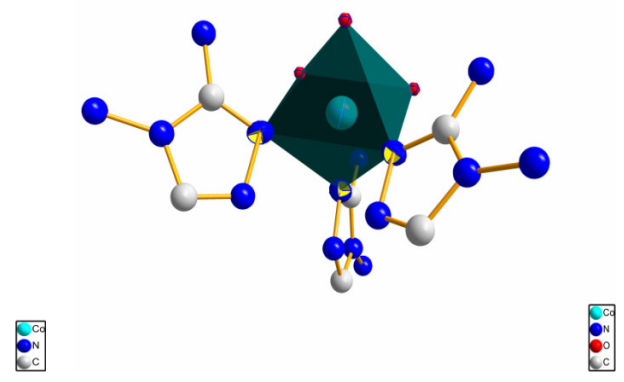

(b) $\mathrm{Co} 3$

Figure 5. The coordination environment of each Co centre in complex 2.

The X-ray diffraction analysis shows that complex 2 involves five metal cobalt cations, twelve DATr molecules, six water molecules and ten perchlorate anions. Although all the cobalt centers are six-fold coordinated, there are some differences in the coordination environments (see Figure 5). The middle three cobalt atoms are connected to six nitrogen atoms of different DATr molecules, while the two-terminal cobalt atoms are surrounded by three nitrogen atoms of three DATr ligands and three oxygen atoms in different water molecules. 
Table 2. Selected bond lengths [̊̊] for complexes 2, 3 and $\mathbf{4}$

\begin{tabular}{|c|c|c|c|c|c|}
\hline \multicolumn{2}{|c|}{ Complex 2} & \multicolumn{2}{|c|}{ Complex 3} & \multicolumn{2}{|c|}{ Complex 4} \\
\hline Bond & $\begin{array}{c}\text { Bond } \\
\text { length } \\
{[\AA]}\end{array}$ & Bond & $\begin{array}{c}\text { Bond } \\
\text { length } \\
{[\AA]} \\
\end{array}$ & Bond & $\begin{array}{c}\text { Bond } \\
\text { length } \\
[\AA]]\end{array}$ \\
\hline $\mathrm{Co}(1)-\mathrm{N}(1)$ & $2.118(7)$ & $\mathrm{Ni}(1)-\mathrm{N}(1)$ & $2.102(11)$ & $\mathrm{Zn}(1)-\mathrm{N}(27)$ & $2.120(7)$ \\
\hline $\mathrm{Co}(1)-\mathrm{N}(1) \# 1$ & $2.118(7)$ & $\mathrm{Ni}(1)-\mathrm{N}(1) \# 1$ & $2.102(11)$ & $\mathrm{Zn}(1)-\mathrm{N}(27) \# 1$ & $2.120(7)$ \\
\hline $\mathrm{Co}(1)-\mathrm{N}(1) \# 2$ & $2.118(7)$ & $\mathrm{Ni}(1)-\mathrm{N}(1) \# 2$ & $2.102(11)$ & $\mathrm{Zn}(1)-\mathrm{N}(32)$ & $2.161(8)$ \\
\hline $\mathrm{Co}(1)-\mathrm{N}(1) \# 3$ & $2.118(7)$ & $\mathrm{Ni}(1)-\mathrm{N}(1) \# 3$ & $2.102(11)$ & $\mathrm{Zn}(1)-\mathrm{N}(32) \# 1$ & $2.161(8)$ \\
\hline $\mathrm{Co}(1)-\mathrm{N}(1) \# 4$ & $2.118(7)$ & $\mathrm{Ni}(1)-\mathrm{N}(1) \# 4$ & $2.102(11)$ & $\mathrm{Zn}(1)-\mathrm{N}(1)$ & $2.165(8)$ \\
\hline $\mathrm{Co}(1)-\mathrm{N}(1) \# 5$ & $2.118(7)$ & $\mathrm{Ni}(1)-\mathrm{N}(1) \# 5$ & 2.102(11) & $\mathrm{Zn}(1)-\mathrm{N}(1) \# 1$ & $2.165(8)$ \\
\hline $\mathrm{Co}(2)-\mathrm{N}(2)$ & $2.145(7)$ & $\mathrm{Ni}(2)-\mathrm{N}(2)$ & $2.096(12)$ & $\mathrm{Zn}(2)-\mathrm{N}(31)$ & $2.128(8)$ \\
\hline $\mathrm{Co}(2)-\mathrm{N}(2 \# 3$ & $2.145(7)$ & $\mathrm{Ni}(2)-\mathrm{N}(2) \# 4$ & $2.096(12)$ & $\mathrm{Zn}(2)-\mathrm{N}(2)$ & $2.129(8)$ \\
\hline $\mathrm{Co}(2)-\mathrm{N}(2) \# 4$ & $2.145(7)$ & $\mathrm{Ni}(2)-\mathrm{N}(2) \# 5$ & $2.096(12)$ & $\mathrm{Zn}(2)-\mathrm{N}(17)$ & $2.144(8)$ \\
\hline $\mathrm{Co}(2)-\mathrm{N}(6)$ & $2.141(8)$ & $\mathrm{Ni}(2)-\mathrm{N}(6)$ & $2.073(12)$ & $\mathrm{Zn}(2)-\mathrm{N}(26)$ & $2.147(8)$ \\
\hline $\mathrm{Co}(2)-\mathrm{N}(6) \# 3$ & $2.141(8)$ & $\mathrm{Ni}(2)-\mathrm{N}(6) \# 4$ & $2.073(12)$ & $\mathrm{Zn}(2)-\mathrm{N}(6)$ & $2.224(8)$ \\
\hline $\mathrm{Co}(2)-\mathrm{N}(6) \# 4$ & $2.141(8)$ & $\mathrm{Ni}(2)-\mathrm{N}(6) \# 5$ & $2.073(12)$ & $\mathrm{Zn}(2)-\mathrm{N}(21)$ & $2.249(9)$ \\
\hline $\mathrm{Co}(3)-\mathrm{N}(7)$ & $2.084(9)$ & $\mathrm{Ni}(3)-\mathrm{N}(7)$ & $2.048(13)$ & $\mathrm{Zn}(3)-\mathrm{N}(11)$ & $1.976(8)$ \\
\hline $\mathrm{Co}(3)-\mathrm{N}(7) \# 3$ & $2.084(9)$ & $\mathrm{Ni}(3)-\mathrm{N}(7) \# 4$ & $2.048(13)$ & $\mathrm{Zn}(3)-\mathrm{N}(22)$ & 1.994(9) \\
\hline $\mathrm{Co}(3)-\mathrm{N}(7) \# 4$ & $2.084(9)$ & $\mathrm{Ni}(3)-\mathrm{N}(7) \# 5$ & $2.048(13)$ & $\mathrm{Zn}(3)-\mathrm{N}(7)$ & $2.013(8)$ \\
\hline $\mathrm{Co}(3)-\mathrm{O}(9)$ & $2.121(8)$ & $\mathrm{Ni}(3)-\mathrm{O}(9)$ & $2.058(11)$ & $\mathrm{Zn}(3)-\mathrm{N}(16)$ & $2.094(8)$ \\
\hline $\mathrm{Co}(3)-\mathrm{O}(9) \# 3$ & $2.121(8)$ & $\mathrm{Ni}(3)-\mathrm{O}(9) \# 4$ & $2.058(11)$ & $\mathrm{Zn}(3)-\mathrm{O}(25)$ & $2.366(9)$ \\
\hline $\mathrm{Co}(3)-\mathrm{O}(9) \# 4$ & $2.121(8)$ & $\mathrm{Ni}(3)-\mathrm{O}(9) \# 5$ & $2.058(11)$ & $\mathrm{N}(1)-\mathrm{C}(1)$ & $1.298(4)$ \\
\hline $\mathrm{N}(1)-\mathrm{N}(2)$ & $1.386(8)$ & $\mathrm{N}(1)-\mathrm{N}(2)$ & $1.425(14)$ & $\mathrm{N}(1)-\mathrm{N}(2)$ & $1.399(3)$ \\
\hline $\mathrm{N}(1)-\mathrm{C}(2)$ & $1.309(11)$ & $\mathrm{N}(1)-\mathrm{C}(2)$ & $1.319(16)$ & $\mathrm{N}(2)-\mathrm{C}(2)$ & $1.328(4)$ \\
\hline $\mathrm{C}(2)-\mathrm{N}(3)$ & $1.359(11)$ & $\mathrm{C}(2)-\mathrm{N}(3)$ & $1.322(16)$ & $\mathrm{N}(3)-\mathrm{N}(4)$ & $1.408(3)$ \\
\hline $\mathrm{N}(3)-\mathrm{C}(1)$ & $1.332(11$ & $\mathrm{N}(3)-\mathrm{C}(1)$ & $1.316(18)$ & $\mathrm{N}(3)-\mathrm{C}(2)$ & $1.357(4)$ \\
\hline $\mathrm{C}(1)-\mathrm{N}(2)$ & $1.386(8)$ & $\mathrm{C}(1)-\mathrm{N}(2)$ & $1.305(18)$ & $\mathrm{N}(3)-\mathrm{C}(1)$ & $1.359(4)$ \\
\hline $\mathrm{N}(3)-\mathrm{N}(4)$ & $1.406(10)$ & $\mathrm{N}(3)-\mathrm{N}(4)$ & $1.422(15)$ & $\mathrm{N}(5)-\mathrm{C}(2)$ & $1.345(4)$ \\
\hline $\mathrm{N}(5)-\mathrm{C}(1)$ & $1.345(11)$ & $\mathrm{N}(5)-\mathrm{C}(1)$ & $1.359(17)$ & & \\
\hline
\end{tabular}

All Co(1)-N have a bond length of 2.118(7) $\AA$. The bond angles around the $\mathrm{Co}(1)$ center are close to the orthogonal requirement (for example: N(1)\#1$\mathrm{Co}(1)-\mathrm{N}(1) \# 2,89.3(3)^{\circ}$; N(1)\#3-Co(1)-N(1)\#4, 89.3(3) ${ }^{\circ}$; N(1)\#2-Co(1)-N(1)\#3, $\left.90.7(3)^{\circ}\right)$ and the angles of $\mathrm{N}-\mathrm{Co}(1)-\mathrm{N}$ (each nitrogen from two contrapuntal DATr ligands) are equal to $180^{\circ}\left(\mathrm{N}(1) \# 1-\mathrm{Co}(1)-\mathrm{N}(1), 180.0(5)^{\circ}, \mathrm{N}(1) \# 2-\right.$ $\left.\operatorname{Co}(1)-\mathrm{N}(1) \# 4,180.0(4)^{\circ}, \mathrm{N}(1) \# 3-\mathrm{Co}(1)-\mathrm{N}(1) \# 5,180.0(4)^{\circ}\right)$, which indicates that the coordination environment of the $\mathrm{Co}(1)$ atom is nearly regular octahedral. Meanwhile, the two Co(2) atoms have the same coordination mode as that of the $\mathrm{Co}(1)$ atom. The bond lengths between $\mathrm{Co}(2)$ and $\mathrm{N} 2$ of the triazole ring range 
from 2.141(8) $\AA$ to 2.145(7) $\AA$. Bond angles of the cobalt atom and the two contra-positioned nitrogen atoms (the angle of $\mathrm{N}-\mathrm{Co}(2)-\mathrm{N}$ is $\left.178.2(3)^{\circ}\right)$ deviate from $180^{\circ}$, resulting in a distorted octahedral structure. But two terminal central atoms are different from the other cobalt atoms. The $\mathrm{Co}(3)-\mathrm{N}$ bond lengths are 2.084(9) Å, while those for Co(3)-O are 2.121(8) A. Moreover, $\mathrm{Co}(3)$ atoms also display distorted octahedral geometry, because of the three atoms $\mathrm{N}(7)$, $\mathrm{Co}(3)$ and $\mathrm{O}(9)$ being not collinear (the angle of $\mathrm{N}(7)-\mathrm{Co}(3)-\mathrm{O}(9)$ is $175.9(3)^{\circ}$ ).

The crystal structure of complex $\mathbf{3}$ is similar to that of complex $\mathbf{2}$, which contains five nickel cations, and the other moieties are the same with complex cobalt (see Figure 7). The three intermediate nickel central atoms exhibit octahedral NiN6 coordination geometry (each nitrogen atom different from six DATr ligands), while the outer two $\mathrm{Ni}$ atoms are ligated with three nitrogen atoms of three 3,4-diamino-1,2,4-triazole molecules and three water oxygen atoms.

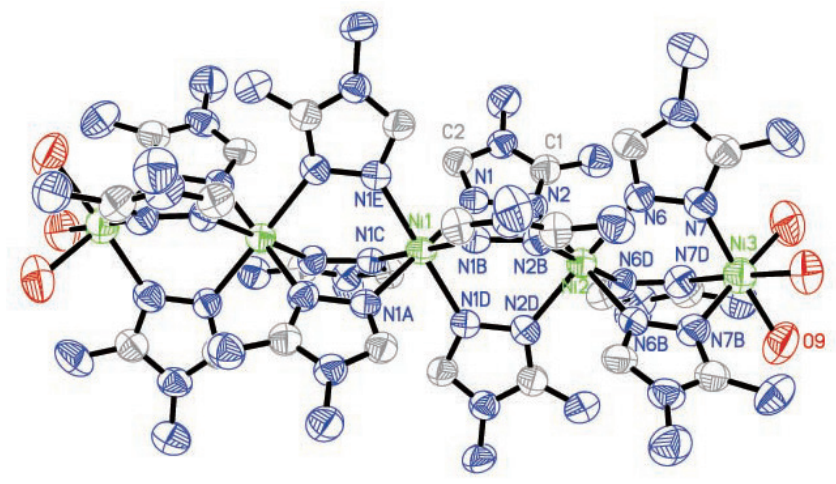

Figure 6. The pentra-nuclear structure of complex 3, thermal ellipsoids drawn at $50 \%$ probability level.

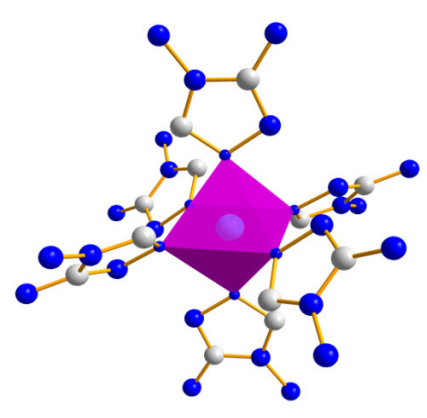

(a) Ni1 and Ni2

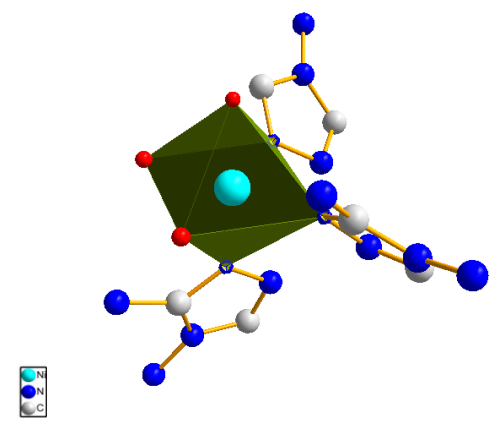

(b) Ni3

Figure 7. The coordination environment of each Ni center in complex $\mathbf{3}$. 
The bond lengths of Ni-N are in the range of 2.048(13) $\AA$ to 2.102(11) $\AA$, while the Ni-O bond distances are 2.058(11) $\AA$, which is shorter than that of the analogous nickel complex [4a]. The bond distances of two adjacent central atoms (Ni-Ni) are 3.7853(2)-3.8203(2) $\AA$. The coordination sphere around all the $\mathrm{Ni}$ (II) atoms except $\mathrm{Ni}(1)$ also shows a distorted octahedron configuration because of the angle of N-Ni-N (two nitrogen atoms of two para-position DATr ligands) and $\mathrm{N}-\mathrm{Ni}-\mathrm{O}$ (one nitrogen atom of triazole ring and one oxygen atom of contrapuntal water molecule) deviating from $180^{\circ}$ (such as the angles of $\mathrm{N}(6)$ $\mathrm{Ni}(2)-\mathrm{N}(2) \# 4, \mathrm{~N}(7)-\mathrm{Ni}(3)-\mathrm{O}(9)$ are $178.9(6)^{\circ}, 177.2(5)^{\circ}$, respectively).

Table 3. Selected bond angles $\left[{ }^{\circ}\right]$ for complexes 2,3 and $\mathbf{4}$

\begin{tabular}{|c|c|c|c|c|c|}
\hline \multicolumn{2}{|l|}{ Complex 2} & \multicolumn{2}{|l|}{ Complex $\mathbf{3}$} & \multicolumn{2}{|l|}{ Complex 4} \\
\hline Bond & $\begin{array}{c}\text { Angle } \\
{\left[^{\circ}\right]}\end{array}$ & Bond & $\begin{array}{c}\text { Angle } \\
{\left[{ }^{\circ}\right]}\end{array}$ & Bond & $\begin{array}{c}\text { Angle } \\
{\left[{ }^{\circ}\right]}\end{array}$ \\
\hline $\mathrm{N}(1)-\mathrm{Co}(1)-\mathrm{N}(1) \# 3$ & $89.3(3)$ & $\mathrm{N}(1)-\mathrm{Ni}(1)-\mathrm{N}(1) \# 4$ & $91.5(5)$ & $\mathrm{N}(27)-\mathrm{Zn}(1)-\mathrm{N}(1)$ & $91.8(3)$ \\
\hline $\mathrm{N}(1)-\mathrm{Co}(1)-\mathrm{N}(1) \# 4$ & $9.3(3)$ & $\mathrm{N}(1)-\mathrm{Ni}(1)-\mathrm{N}(1) \# 5$ & $91.5(5)$ & $\mathrm{N}(27)-\mathrm{Zn}(1)-\mathrm{N}(32)$ & $90.4(3)$ \\
\hline $\mathrm{N}(1) \# 3-\mathrm{Co}(1)-\mathrm{N}(1) \# 4$ & $89.3(3)$ & $\mathrm{N}(1) \# 4-\mathrm{Ni}(1)-\mathrm{N}(1) \# 5$ & $91.5(5)$ & $\mathrm{N}(32)-\mathrm{Zn}(1)-\mathrm{N}(1)$ & $89.3(3)$ \\
\hline $\mathrm{N}(1) \# 1-\mathrm{Co}(1)-\mathrm{N}(1) \# 2$ & $89.3(3)$ & $\mathrm{N}(1) \# 1-\mathrm{Ni}(1)-\mathrm{N}(1) \# 2$ & $91.5(5)$ & $\mathrm{N}(27) \# 1-\mathrm{Zn}(1)-\mathrm{N}(1) \# 1$ & $91.8(3)$ \\
\hline $\mathrm{N}(1) \# 1-\mathrm{Co}(1)-\mathrm{N}(1) \# 5$ & $89.3(3)$ & $\mathrm{N}(1) \# 2-\mathrm{Ni}(1)-\mathrm{N}(1) \# 3$ & $91.5(5)$ & $\mathrm{N}(27) \# 1-\mathrm{Zn}(1)-\mathrm{N}(32) \# 1$ & $90.4(3)$ \\
\hline $\mathrm{N}(1) \# 2-\mathrm{Co}(1)-\mathrm{N}(1) \# 5$ & $89.3(3)$ & $\mathrm{N}(1) \# 1-\mathrm{Ni}(1)-\mathrm{N}(1) \# 3$ & $91.5(5)$ & $\mathrm{N}(32) \# 1-\mathrm{Zn}(1)-\mathrm{N}(1) \# 1$ & $89.3(3)$ \\
\hline $\mathrm{N}(1) \# 1-\mathrm{Co}(1)-\mathrm{N}(1)$ & $180.0(5)$ & $\mathrm{N}(1)-\mathrm{Ni}(1)-\mathrm{N}(1$ & $180.0(12)$ & $\mathrm{N}(27)-\mathrm{Zn}(1)-\mathrm{N}($ & $180.0(1)$ \\
\hline $\mathrm{N}(1) \# 2-\mathrm{Co}(1)-\mathrm{N}(1) \# 4$ & $180.0(4)$ & $\mathrm{N}(1) \# 3-\mathrm{Ni}(1)-\mathrm{N}(1) \# 4$ & $180.0(8)$ & $\mathrm{N}(32)-\mathrm{Zn}(1)-\mathrm{N}(32) \# 1$ & $180.0(2)$ \\
\hline $\mathrm{N}(1) \# 3-\mathrm{Co}(1)-\mathrm{N}(1) \# 5$ & $180.0(4)$ & $\mathrm{N}(1) \# 2-\mathrm{Ni}(1)-\mathrm{N}($ & \begin{tabular}{|l|}
$180.0(7)$ \\
\end{tabular} & $\mathrm{N}(1)-\mathrm{Zn}(1)-\mathrm{N}($ & $180.0(4)$ \\
\hline $\mathrm{N}(2) \# 3-\mathrm{Co}(2)-\mathrm{N}(2) \# 4$ & $91.8(3)$ & $\mathrm{N}(2)-\mathrm{Ni}(2)-\mathrm{N}(2$ & $92.2(5)$ & $\mathrm{N}(6)-\mathrm{Zn}(2)-\mathrm{N}$ & $86.4(3)$ \\
\hline $\mathrm{N}(2) \# 3-\mathrm{Co}(2)-\mathrm{N}(2)$ & $91.8(3)$ & $\mathrm{N}(2) \# 4-\mathrm{Ni}(2)-\mathrm{N}(2) \# 5$ & $92.2(5)$ & $\mathrm{N}(17)-\operatorname{Zn}(2)-\mathrm{N}$ & $93.4(3)$ \\
\hline $\mathrm{N}(2) \# 4-\mathrm{Co}(2)-\mathrm{N}(2)$ & $91.8(3)$ & $\mathrm{N}(2) \# 4-\mathrm{Ni}(2)-\mathrm{N}$ & $92.2(5)$ & $\mathrm{N}(17)-\mathrm{Zn}(2)-\mathrm{I}$ & $85.6(3)$ \\
\hline $\mathrm{N}(6)-\mathrm{Co}(2)-\mathrm{N}($ & $89.8(3)$ & $\mathrm{N}(6)-\mathrm{Ni}(2)-\mathrm{I}$ & $90.8(5)$ & $\mathrm{N}(31)-\mathrm{Zn}(2)-\mathrm{N}$ & $92.5(3)$ \\
\hline $\mathrm{N}(6)-\mathrm{Co}(2)-\mathrm{N}($ & $89.8(3)$ & $\mathrm{N}(6)-\mathrm{Ni}(2)-\mathrm{N}$ & $90.8(5)$ & $\mathrm{N}(31)-\operatorname{Zn}(2)-\mathrm{N}$ & $91.2(3)$ \\
\hline $\mathrm{N}(6) \# 3-\mathrm{Co}(2)$ & $89.8(3)$ & $\mathrm{N}(6) \# 4-\mathrm{Ni}(2$ & \begin{tabular}{|l|}
$90.8(5)$ \\
\end{tabular} & $\mathrm{N}(2)-\mathrm{Zn}(2)-\mathrm{N}$ & 95.4(3) \\
\hline $\mathrm{N}(6) \# 4-\mathrm{Co}(2)-\mathrm{N}(2) \# 3$ & $178.2(3)$ & $\mathrm{N}(6)-\mathrm{Ni}(2)-\mathrm{N}$ & \begin{tabular}{|l|}
$178.9(6)$ \\
\end{tabular} & $\mathrm{N}(31)-\mathrm{Zn}(2)-\mathrm{N}$ & $177.9(3)$ \\
\hline $\mathrm{N}(6)-\mathrm{Co}(2)-\mathrm{N}(2) \# 4$ & $178.2(3)$ & $\mathrm{N}(6) \# 5-\mathrm{Ni}(2)-\mathrm{N}(2)$ & 178.9(5) & $\mathrm{N}(26)-\mathrm{Zn}(2)-\mathrm{N}(6)$ & 166.1(3) \\
\hline $\mathrm{N}(6) \# 3-\mathrm{Co}(2)-\mathrm{N}(2)$ & $178.2(3)$ & $\mathrm{N}(6) \# 4-\mathrm{Ni}(2)-\mathrm{N}(2) \# 5$ & $178.9(5)$ & $\mathrm{N}(2)-\mathrm{Zn}(2)-\mathrm{N}(21)$ & $178.0(3)$ \\
\hline $\mathrm{N}(7) \# 3-\mathrm{Co}(3)-\mathrm{N}(7)$ & $92.9(3)$ & $\mathrm{N}(7) \# 5-\mathrm{Ni}(3)-\mathrm{N}(7)$ & $91.9(6)$ & $\mathrm{N}(11)-\mathrm{Zn}(3)-\mathrm{N}(22)$ & $129.8(3)$ \\
\hline $\mathrm{N}(7)-\mathrm{Co}(3)-\mathrm{N}(7) \# 4$ & $92.9(3)$ & $\mathrm{N}(7) \# 4-\mathrm{Ni}(3)-\mathrm{N}(7)$ & $91.9(6)$ & $\mathrm{N}(11)-\mathrm{Zn}(3)-\mathrm{N}(7)$ & $120.6(4)$ \\
\hline $\mathrm{N}(7) \# 3-\mathrm{Co}(3)-\mathrm{N}(7) \# 4$ & $92.9(3)$ & $\mathrm{N}(7) \# 5-\mathrm{Ni}(3)-\mathrm{N}(7) \# 4$ & $91.9(6)$ & $\mathrm{N}(22)-\mathrm{Zn}(3)-\mathrm{N}(7)$ & 102.9(3) \\
\hline $\mathrm{O}(9) \# 3-\mathrm{Co}(3)-\mathrm{O}(9) \# 4$ & $87.1(4)$ & $\mathrm{O}(9) \# 5-\mathrm{Ni}(3)-\mathrm{O}(9) \# 4$ & $88.6(5)$ & $\mathrm{N}(16)-\mathrm{Zn}(3)-\mathrm{O}(25)$ & $176.6(3)$ \\
\hline $\mathrm{N}(7)-\mathrm{Co}(3)-\mathrm{O}(9) \# 3$ & $89.7(3)$ & $\mathrm{N}(7) \# 4-\mathrm{Ni}(3)-\mathrm{O}(9) \# 5$ & $90.9(5)$ & $\mathrm{N}(11)-\mathrm{Zn}(3)-\mathrm{N}(22)$ & $129.8(3)$ \\
\hline $\mathrm{N}(7) \# 4-\mathrm{Co}(3)-\mathrm{O}(9) \# 3$ & $90.3(3)$ & $\mathrm{N}(7)-\mathrm{Ni}(3)-\mathrm{O}(9) \# 5$ & $88.6(5)$ & $\mathrm{N}(22)-\mathrm{Zn}(3)-\mathrm{N}(16)$ & $98.0(3)$ \\
\hline $\mathrm{N}(7)-\mathrm{Co}(3)-\mathrm{O}(9)$ & $175.9(3)$ & $\mathrm{N}(7)-\mathrm{Ni}(3)-\mathrm{O}(9)$ & $177.2(5)$ & $\mathrm{N}(7)-\mathrm{Zn}(3)-\mathrm{N}(16)$ & $95.1(3)$ \\
\hline $\mathrm{N}(7) \# 3-\mathrm{Co}(3)-\mathrm{O}(9) \# 3$ & $175.9(3)$ & $\mathrm{N}(7) \# 4-\mathrm{Ni}(3)-\mathrm{O}(9) \# 4$ & \begin{tabular}{|l|}
$177.2(5)$ \\
\end{tabular} & & \\
\hline $\mathrm{N}(7) \# 4-\mathrm{Co}(3)-\mathrm{O}(9) \# 4$ & $175.9(3)$ & $\mathrm{N}(7) \# 5-\mathrm{Ni}(3)-\mathrm{O}(9) \# 5$ & $177.2(5)$ & & \\
\hline
\end{tabular}

The molecular structure of complex 4 is slightly different to those of complexes 2 and $\mathbf{3}$, being composed of five central zinc atoms, fourteen DATr ligands, four 
water molecules (two lattice molecules and two coordinated molecules) and ten perchlorate anions. The metal atoms have different coordination situations (see Figure 9), including coordination numbers and coordination sites. The three middle zinc atoms are six-fold-coordinated (the same as for complexes $\mathbf{2}$ and $\mathbf{3}$ ), but two terminal central atoms are penta-coordinated.

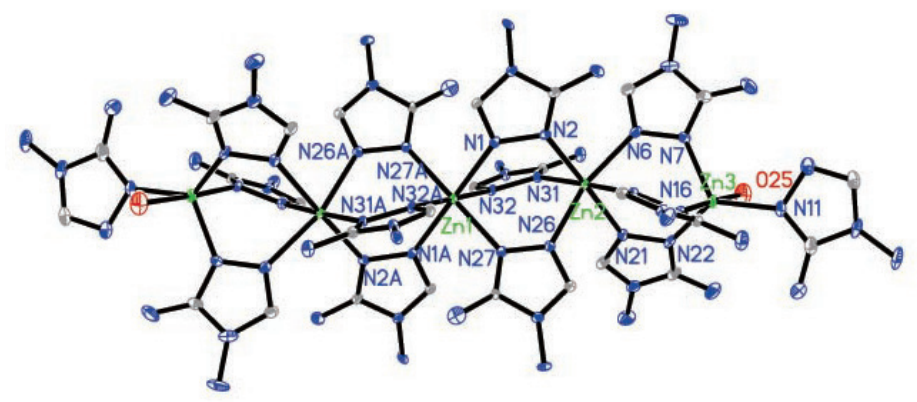

Figure 8. The pentra-nuclear structure of complex 4, thermal ellipsoids drawn at $50 \%$ probability level.

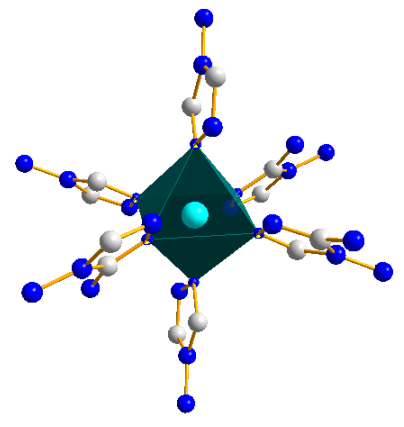

(a) Zn1 and Zn2

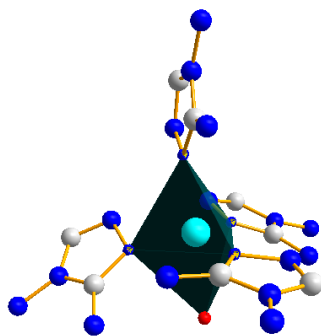

(b) $\mathrm{Zn} 3$

Figure 9. The coordination environment of each Ni center in complex 4.

It is observed that the bond lengths of $\mathrm{M}-\mathrm{N}(\mathrm{M}=\mathrm{Zn}(1), \mathrm{Zn}(2))$ is not the same (2.165(8)-2.249(9) $\AA$ ) and N-M-N (M = Zn(1), Zn(2)) bond angles (each nitrogen atom from two contra-position DATr molecules) are not equal to $180^{\circ}$, which contributes to the distorted octahedron. The torsion angle of $\mathrm{N}(7)-\mathrm{Zn}(3)$ $\mathrm{N}(11)-\mathrm{N}(12)$ is close to $0^{\circ}\left(0.6(9)^{\circ}\right)$, which demonstrates that the metal $\mathrm{Zn}(3)$ atom as well as the other three nitrogen atoms $(\mathrm{N}(7), \mathrm{N}(11)$ and $\mathrm{N}(12)$ occupies the same plane. The coordination environment of the two outer $\mathrm{Zn}$ centers generates similar trigonal bipyramid geometry [16]. What's more, the other 
coordinated nitrogen atom and oxygen atom comprise the axial position (the angle of $\mathrm{N}(16)-\mathrm{Zn}(3)-\mathrm{O}(25)$ is $\left.176.6(3)^{\circ}\right)$. The $\mathrm{Zn}(1) \ldots \mathrm{Zn}(2)$ distance $(3.7950(3) \AA)$ is shorter than that of $\mathrm{Zn}(2) \ldots \mathrm{Zn}(3)$ (3.6694(3) $\AA$ ), due to the different coordination environments of the central atoms.

Table 4. Hydrogen bond distances $[\AA]$ and angles $\left[{ }^{\circ}\right]$ for complexes $\mathbf{2 , 3}$, and 4

\begin{tabular}{|c|c|c|c|c|c|}
\hline D-H...A & $\begin{array}{c}\mathrm{d}(\mathrm{D}-\mathrm{H}) \\
{[\AA]}\end{array}$ & $\begin{array}{c}\mathrm{d}(\mathrm{H} \ldots \mathrm{A}) \\
{[\AA]}\end{array}$ & $\begin{array}{c}\mathrm{d}(\mathrm{D} \ldots \mathrm{A}) \\
{[\because \AA]}\end{array}$ & $\begin{array}{c}<(\mathrm{DHA}) \\
{\left[{ }^{\circ}\right]}\end{array}$ & Symmetry code \\
\hline \multicolumn{6}{|l|}{ Complex 2} \\
\hline N4-H4B...O8A & 0.860 & 2.578 & 3.322 & 145.32 & $\mathrm{y},-\mathrm{x}+\mathrm{y},-\mathrm{z}$ \\
\hline N4-H4B...N10 & 0.860 & 2.613 & 3.220 & 128.56 & $x-y+2 / 3, x+1 / 3,-z+1 / 3$ \\
\hline N5-H5A...O1 & 0.860 & 2.479 & 2.996 & 119.43 & \\
\hline N5-H5A...N6 & 0.860 & 2.605 & 3.201 & 127.39 & $-x+y,-x, z$ \\
\hline N10-H10A...O9 & 0.860 & 2.580 & 3.173 & 127.07 & $-x+y,-x, z$ \\
\hline O9-H9E...O1 & 0.850 & 2.133 & 2.923 & 154.46 & $-x+y,-x, z$ \\
\hline O9-H9E...N7 & 0.850 & 2.540 & 2.964 & 111.94 & $-x+y,-x, z$ \\
\hline O9-H9F...O8A & 0.850 & 2.259 & 3.045 & 154.01 & $x-1 / 3, y-2 / 3, z+1 / 3$ \\
\hline O9-H9F...Cl2 & 0.850 & 2.989 & 3.733 & 147.41 & $x-1 / 3, y-2 / 3, z+1 / 3$ \\
\hline \multicolumn{6}{|l|}{ Complex $\mathbf{3}$} \\
\hline N4-H4B...O8A & 0.860 & 2.559 & 3.303 & 145.25 & $\mathrm{y},-\mathrm{x}+\mathrm{y},-\mathrm{z}$ \\
\hline N4-H4B...O3A & 0.860 & 2.602 & 3.091 & 117.19 & $-x+y+1 / 3,-x+2 / 3, z-1 / 3$ \\
\hline N5-H5A...O1 & 0.860 & 2.478 & 2.974 & 117.43 & \\
\hline N5-H5A...N6 & 0.860 & 2.585 & 3.181 & 127.31 & $-x+y,-x, z$ \\
\hline N10-H10A...O9 & 0.860 & 2.397 & 3.127 & 143.08 & $-x+y,-x, z$ \\
\hline O9-H9E...N10 & 0.850 & 2.374 & 2.900 & 120.57 & $-x+y,-x, z$ \\
\hline O9-H9F...O8A & 0.850 & 2.286 & 3.134 & 175.85 & $x-1 / 3, y-2 / 3, z+1 / 3$ \\
\hline \multicolumn{6}{|l|}{ Complex 4} \\
\hline N4-H4A...O12A & 0.860 & 2.405 & 3.097 & 137.87 & $\mathrm{x}-1, \mathrm{y}+1, \mathrm{z}$ \\
\hline N9-H9A...O17 & 0.860 & 2.068 & 2.928 & 178.88 & $-x,-y+2,-z+1$ \\
\hline N10-H10B...O18 & 0.860 & 2.390 & 3.145 & 146.85 & $\mathrm{x}-1, \mathrm{y}, \mathrm{z}-1$ \\
\hline N15-H15A...O22 & 0.860 & 2.139 & 2.952 & 157.47 & $-\mathrm{x}+1,-\mathrm{y}+1,-\mathrm{z}+1$ \\
\hline N15-H15B...N25 & 0.860 & 2.400 & 3.143 & 144.94 & $-x+1,-y+1,-z$ \\
\hline N19-H19A...N9 & 0.860 & 2.665 & 3.149 & 116.85 & $\mathrm{x}+1, \mathrm{y}, \mathrm{z}$ \\
\hline N20-H20A...O18 & 0.956 & 2.528 & 3.087 & 117.42 & $-x+1,-y+2,-z+1$ \\
\hline N20-H20A...N11 & 0.956 & 2.536 & 3.302 & 137.16 & \\
\hline N24-H24A...Cl1 & 0.860 & 2.926 & 3.739 & 158.49 & $-\mathrm{x}+1,-\mathrm{y}+1,-\mathrm{z}+1$ \\
\hline $\mathrm{O} 25-\mathrm{H} 25 \mathrm{C} \ldots \mathrm{O} 26$ & 0.850 & 2.330 & 2.744 & 110.37 & $\mathrm{x}, \mathrm{y}, \mathrm{z}-1$ \\
\hline $\mathrm{O} 25-\mathrm{H} 25 \mathrm{D} \ldots \mathrm{N} 25$ & 0.850 & 2.589 & 3.028 & 113.32 & \\
\hline $\mathrm{O} 26-\mathrm{H} 26 \mathrm{~B} \ldots \mathrm{Cl} 2$ & 0.850 & 2.981 & 3.671 & 139.80 & $-\mathrm{x}+1,-\mathrm{y}+1,-\mathrm{z}+1$ \\
\hline
\end{tabular}


The separate penta-nuclear molecules of the three complexes are aligned into 3D supra-molecular network structures through large amounts of hydrogenbonding interactions including intra-molecular and intermolecular. Detailed hydrogen bonds of complexes 2-4 are listed in Table 4. In complex 2, intramolecular hydrogen bonds occur between the oxygen atoms of the perchlorate anions and nitrogen atoms of the amino groups and triazole rings. What's more, perchlorate anions mainly form intermolecular $\mathrm{H}$-bonding interactions to link two adjacent penta-nuclear units. In complex $\mathbf{3}$, hydrogen bonds between coordinated water molecules, amino groups and perchlorate anions are observed, but the chlorine atoms of the perchlorate anions are not found as acceptors in hydrogen bonds. The connecting mode of two neighboring five-nuclear units in complex 3 through intermolecular H-bonding interaction is similar to that of complex 2 . In complex 4 , because it contains lattice water molecules, hydrogen-bonding interactions are rather more complicated compared to the other coordination compounds. The intra-molecular hydrogen bonds appear among crystal water molecules, perchlorate anions, amino groups and triazole rings. Furthermore, apart from perchlorate anions, the amino groups of DATr ligands in the contiguous penta-nuclear units also participate in inter-molecular hydrogen-bonding interactions.

\subsection{Thermal Stabilities}

The thermal decomposition behavior of the complexes has been investigated using differential scanning calorimetry (DSC). The DSC curves of the four complexes for a heating rate of $5^{\circ} \mathrm{C} \cdot \mathrm{min}^{-1}$ are shown in Figure 8. Endothermic peaks of most of the title coordination compounds are not observed in the DSC plots except for complex 3 . The decomposition temperatures of complexes 1-4 are above $300{ }^{\circ} \mathrm{C}$ based on their onset DSC peaks. The thermal stabilities of the four complexes are higher than the criterion of $200{ }^{\circ} \mathrm{C}$ for "green" metal energetic materials [17]. Therefore, these metal complexes with the DATr ligand are sufficiently thermally stable to be energetic materials. The decomposition process of complex 1 occurs in the temperature range of $289.2-382.3{ }^{\circ} \mathrm{C}$ with one main exothermic peak. For complex 2, an exothermic stage is seen with a sharp peak temperature at $305.8^{\circ} \mathrm{C}$. It is observed from the DSC curve of complex 3 that a wide exothermic process appears at temperatures between $269.7^{\circ} \mathrm{C}$ and $390.8^{\circ} \mathrm{C}$. For complex 4, a small endothermic process is found at 293.5-314.3 ${ }^{\circ} \mathrm{C}$ which can indicate a phase transition, and there is an exothermic stage in the range of $320.1-370.2{ }^{\circ} \mathrm{C}$, with peak temperature at $337.4^{\circ} \mathrm{C}$. Their thermal stabilities increase in the sequence of compounds: $\mathbf{2}<\mathbf{1}<\mathbf{4}<\mathbf{3}$. 


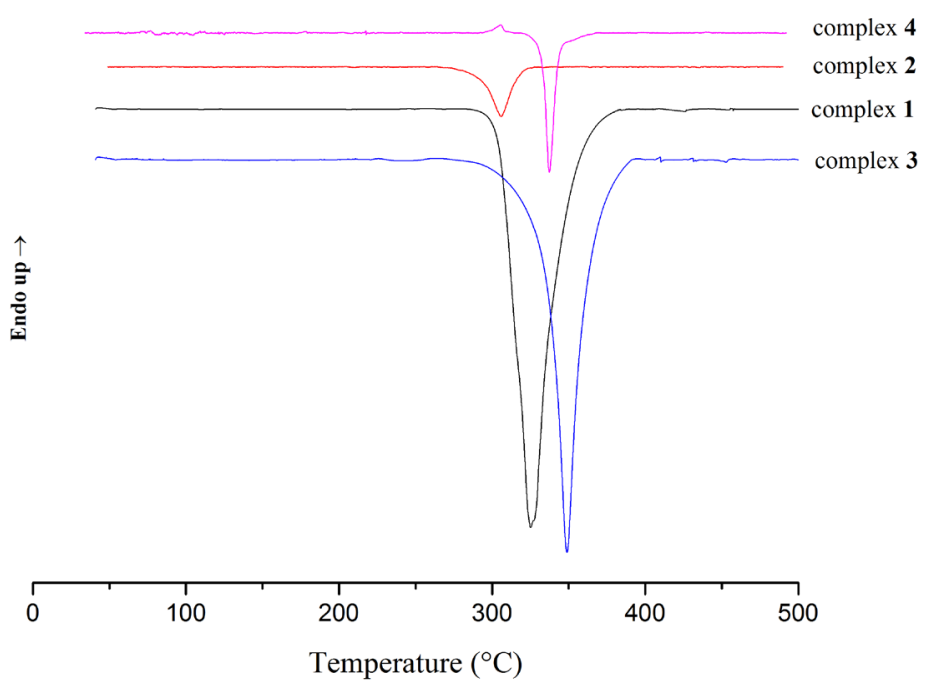

Figure 10. The DSC curve of the four complexes for a heating rate of $5^{\circ} \mathrm{C} \cdot \mathrm{min}^{-1}$.

In general, energy of combustion is a significant parameter to assess the energetic properties of new complexes. The energies of combustion of the four complexes are surveyed in Table 5. Constant volume energies of combustion $\left(Q_{v}\right)$ were measured by oxygen bomb calorimetry in an oxygen atmosphere. The other physicochemical properties of the title complexes are also listed in Table 5. The nitrogen contents of all the coordination compounds are over $30 \%$ by weight. Furthermore, these complexes can release a large amount of heat in the combustion process.

Table 5. Physicochemical properties of complexes 1-4

\begin{tabular}{|l|c|c|c|c|}
\hline & Complex 1 & Complex 2 & Complex 3 & Complex 4 \\
\hline $\mathrm{T}_{\mathrm{p}}\left[{ }^{\circ} \mathrm{C}\right]^{\mathrm{a}}$ & 347.7 & 328.6 & 361.9 & 350.9 \\
\hline $\mathrm{N}[\%]^{\mathrm{b}}$ & 32.75 & 32.49 & 32.51 & 35.26 \\
\hline$\Omega[\%]^{\mathrm{c}}$ & -23.71 & -23.51 & -23.52 & -29.34 \\
\hline $\mathrm{E}\left[\mathrm{kJ} \cdot \mathrm{mol}^{-1}\right]^{\mathrm{d}}$ & 101.65 & 112.80 & 183.00 & 175.90 \\
\hline $\mathrm{Q}_{\mathrm{v}}\left[\mathrm{MJ} \cdot \mathrm{kg}^{-1}\right]^{\mathrm{e}}$ & 8.91 & 9.51 & 8.73 & 8.24 \\
\hline
\end{tabular}

${ }^{\text {a }}$ Thermal decomposition/ DSC main exothermic peak at the heat rate of $10^{\circ} \mathrm{C} \cdot \mathrm{min}^{-1}$;

${ }^{\mathrm{b}}$ Nitrogen content; ${ }^{\mathrm{c}}$ Oxygen balance; ${ }^{\mathrm{d}}$ Activation energy; ${ }^{\mathrm{e}}$ Constant volume energy of combustion.

In the research presented in this paper, Kissinger's methods [18] and Ozawa's methods [19] were used to determine the Arrhenius equation for a given material. 
The Kissinger (Equation a) and Ozawa (Equation b) equations are as follows:

$$
\begin{aligned}
& \ln \beta / \mathrm{T}_{\mathrm{p}}^{2}=\ln \left[\mathrm{RA} / \mathrm{E}_{\mathrm{a}}\right]-\mathrm{E}_{\mathrm{a}} /\left(\mathrm{RT}_{\mathrm{p}}\right) \\
& \lg \beta=\lg [\mathrm{AEa} / \mathrm{RG}(\alpha)]-2.315-0.4567 \mathrm{E}_{\mathrm{a}} /\left(\mathrm{RT}_{\mathrm{p}}\right)
\end{aligned}
$$

$T_{\mathrm{p}}$ is the temperature $\left({ }^{\circ} \mathrm{C}\right)$ at which the first exothermic peak occurs in the DSC curve; $A$ is the pre-exponential factor $\left[\mathrm{s}^{-1}\right] ; E_{a}$ is the apparent activation energy $\left[\mathrm{kJ} \cdot \mathrm{mol}^{-1}\right] ; R$ is the gas constant $\left(8.314 \mathrm{~J} \cdot \mathrm{K}^{-1} \cdot \mathrm{mol}^{-1}\right) ; \beta$ is the linear heating rate $\left[\mathrm{K} \cdot \mathrm{min}^{-1}\right]$; and $G(\alpha)$ is the reaction-mechanism function.

Based on the first exothermic peaks measured with four different heating rates of $5,10,15$ and $20{ }^{\circ} \mathrm{C} \cdot \mathrm{min}^{-1}$, Kissinger's and Ozawa's methods were applied to investigate the kinetic parameters of the title complexes. From these data, the apparent activation energies $E_{\mathrm{K}}$ and $E_{\mathrm{O}}$ ( $E$ is the average value of $E_{\mathrm{K}}$ and $E_{\mathrm{O}}$ ), pre-exponential factor $A_{\mathrm{K}}$, linear correlation coefficients $R_{\mathrm{K}}$ and $R_{\mathrm{O}}$ were determined and are listed in Table 6.

Table 6. The temperature of the first main exothermic peak for different

\begin{tabular}{|c|c|c|c|c|c|}
\hline & & Complex 1 & Complex 2 & Complex 3 & Complex 4 \\
\hline & 5 & 325.8 & 305.8 & 348.8 & 337.4 \\
\hline $\mathrm{T}_{\mathrm{p}}\left[{ }^{\circ} \mathrm{C}\right]$ & 10 & 347.7 & 328.6 & 361.9 & 350.9 \\
\hline value & 15 & 353.5 & 333.5 & 368.9 & 357.1 \\
\hline & 20 & 367.3 & 337.4 & 372.1 & 361.1 \\
\hline Kissinger's m & & & & & \\
\hline $\mathrm{E}_{\mathrm{K}}\left[\mathrm{kJ} \cdot \mathrm{mol}^{-1}\right]$ & & 99.2 & 110.8 & 182.5 & 175.3 \\
\hline $\ln A_{k}$ & & 6.09 & 7.50 & 12.99 & 12.66 \\
\hline $\mathrm{R}_{\mathrm{K}}$ & & 0.9863 & 0.9646 & 0.9952 & 0.9958 \\
\hline Ozawa's meth & & & & & \\
\hline$E_{\mathrm{O}}\left[\mathrm{kJ} \cdot \mathrm{mol}^{-1}\right]$ & & 104.1 & 114.8 & 183.5 & 176.5 \\
\hline$R_{\mathrm{O}}$ & & 0.9887 & 0.9698 & 0.9957 & 0.9962 \\
\hline
\end{tabular}
heating rates and chemical kinetics parameters

Accordingly, the Arrhenius equation for the coordination compounds can be expressed as Equations 1, 2, 3 and 4:

$$
\begin{aligned}
& \ln \mathrm{k}=6.09-101.65 \times 10^{3} /(\mathrm{RT}) \\
& \ln \mathrm{k}=7.50-112.80 \times 10^{3} /(\mathrm{RT}) \\
& \ln \mathrm{k}=12.99-183.00 \times 10^{3} /(\mathrm{RT}) \\
& \ln \mathrm{k}=12.66-175.90 \times 10^{3} /(\mathrm{RT})
\end{aligned}
$$


The above equations can be used to estimate the rate constants of the initial thermal decomposition process of the four complexes. The activation energy of the nickel complex is larger than that of the other complexes.

\subsection{Sensitivities}

In order to evaluate the possibility of using the four compounds as energetic materials, the impact and friction sensitivities for the initial safety testing were measured according to BAM methods [20] with the "BAM Fallhammer" and "BAM friction tester". The results are summarized in Table 7. In general, the perchlorate complexes were more sensitive to external stimuli than the corresponding nitrates [1c, 21].

Table 7. The sensitivities testing results for four complexes

\begin{tabular}{|l|c|c|c|c|}
\hline & $\mathbf{1}$ & $\mathbf{2}$ & $\mathbf{3}$ & $\mathbf{4}$ \\
\hline Impact sensitivity [J] & $>40$ & 1.82 & 2.68 & $>40$ \\
\hline Friction sensitivity [N] & 128 & 84 & 60 & 216 \\
\hline
\end{tabular}

Complex $\mathbf{2}$ is very sensitive towards impact and complex $\mathbf{3}$ also shows high sensitivity. However, complexes $\mathbf{1}$ and $\mathbf{4}$ are both insensitive to impact. Complex $\mathbf{2}$ was found to be more impact sensitive than lead azide while complex $\mathbf{3}$ is similar to lead azide [22]. According to the UN Recommendations on the Transport of Dangerous Goods [23], complexes $\mathbf{2 , 3}$ are classified as very sensitive towards impact but complexes 1, $\mathbf{4}$ are classified as insensitive towards impact. In addition, complexes $\mathbf{1 , 2}$ and $\mathbf{4}$ are sensitive to friction, and complex $\mathbf{3}$ is very sensitive towards friction. However, the four complexes aren't more sensitive against friction than that of lead azide [3g]. On the whole, it is found for safety sensitivities that complex $\mathbf{2}$ is the most sensitive towards external stimuli of the four complexes but complex $\mathbf{4}$ is relatively insensitive.

\section{Conclusions}

3,4-diamino-1,2,4-triazole was coordinated with several transition metal $(\mathrm{M}=\mathrm{Mn}, \mathrm{Co}, \mathrm{Ni}, \mathrm{Zn})$ perchlorates to form energetic complexes. All the complexes were characterized by elemental analysis, infrared spectroscopy and differential scanning calorimetry. Single-crystal structures of complexes 2-4 were obtained. All three crystal structures mainly consist of penta-nuclear units, and developed 3D supra-molecular network constructions through extensive hydrogen-bond interactions. DSC results suggest that all four complexes 
have good thermal stabilities (decompose above $300^{\circ} \mathrm{C}$ ) and the Co complex has the highest decomposition temperature. Non-isothermal kinetic analysis indicates that the Arrhenius equations of complexes 1-4 can be expressed as follows: $\ln k=6.09-101.65 \times 10^{3} /(R T)$ (for 1), $\ln k=7.50-112.80 \times 10^{3} /(R T)$ (for complex 2), $\ln k=12.99-183.00 \times 10^{3} /(R T)$ (for complex 3) and $\ln k=12.66-175.90 \times 10^{3} /(R T)$ (for complex 4). Furthermore, the combustion performance and other energetic properties were also studied. All complexes containing energetic counterions show different sensitivities towards mechanical stimulations. More importantly, the cobalt and nickel complexes are extremely sensitive towards impact stimulation, which are similar to that of lead azide, whereas complexes 1, 4 are insensitive against impact. Besides, most of the title compounds are sensitive to friction and the nickel complex is very sensitive towards friction. However, it is found that all the coordination compounds are less sensitive towards friction than lead azide. Therefore, in conclusion complexes 2 and $\mathbf{3}$ are potential primary explosives with high thermal stabilities (complex $\mathbf{2}$ decomposes at $328.6^{\circ} \mathrm{C}$ and complex $\mathbf{3}$ decomposes at $361.9^{\circ} \mathrm{C}$ ) to replace toxic lead azide (which decomposes at $315^{\circ} \mathrm{C}$ ).

\section{Acknowledgments}

We gratefully acknowledge financial support from the National Natural Science Foundation of China (10776002), and the project of State Key Laboratory of Science and Technology (No. ZDKT12-03 \& YBKT16-04).

\section{References}

[1] a) Klapötke T.M., Petermayer C., Piercey D.G., Stierstorfer J., 1,3-Bis(nitroimido)1,2,3-triazolate Anion, the N-Nitroimide Moiety, and the Strategy of Alternating Positive and Negative Charges in the Design of Energetic Materials, J. Am. Chem. Soc., 2012, 134, 20827-20836; b) Kumar A.S., Ghule V.D., Subrahmanyam S., Sahoo A.K., Synthesis of Thermally Stable Energetic 1,2,3-Triazole Derivatives, Chem. Eur. J., 2013, 19, 509-518; c) Joas M., Klapötke T.M., Stierstorfer J., Szimhardt N., Synthesis and Characterization of Various Photosensitive Copper(II) Complexes with 5-(1-Methylhydrazinyl)-1H-tetrazole as Ligand and Perchlorate, Nitrate, Dinitramide, and Chloride as Anions, Chem. Eur. J., 2013, 19, 9995-10003.

[2] a) Tao G.H., Parrish D.A., Shreeve J.M., Nitrogen-rich 5-(1-Methylhydrazinyl) tetrazole and its Copper and Silver Complexes, Inorg. Chem., 2012, 51, 530512; b) Tao G.H., Twamley B., Shreeve J.M., Energetic Nitrogen-rich Cu(II) and Cd(II) 5,5'-Azobis(tetrazolate) Complexes, Inorg. Chem., 2009, 48, 9918-9923; c) Zhao H., Qu Z.-R., Ye H.-Y., Xiong R.-G., In situ Hydrothermal Synthesis of 
Tetrazole Coordination Polymers with Interesting Physical Properties, Chem. Soc. Rev, 2008, 37, 84-100.

[3] a) Singh R.P., Verma R.D., Meshri D.T., Shreeve J.M., Energetic NitrogenRich Salts and Ionic Liquids. Angew. Chem., Int. Ed., 2006, 45, 3584-3601; b) Fischer N., Joas M., Klapötke T.M., Stierstorfer J., Transition Metal Complexes of 3-Amino-1-nitroguanidine as Laser Ignitible Primary Explosives: Structures and Properties, Inorg. Chem., 2013, 52, 13791-13802; c) Sivabalan R., Anniyappan M., Pawar S.J., Talawar M.B., Gore G.M., Venugopalan S., Gandhe B.R., Synthesis, Characterization and Thermolysis Studies on Triazole and Tetrazole Based High Nitrogen Content High Energy Materials, J. Hazard. Mater, 2006, 137, 672680; d) Talawar M.B., Sivabalan R., Mukundan T., Muthurajan H., Sikder A.K., Gandhe B.R., Rao A.S., Environmentally Compatible Next Generation Green Energetic Materials (GEMs), J. Hazard. Mater, 2009, 161, 589-607; e) Cui Y., Zhang J., Zhang T., Yang L., Zhang J., Hu X., Synthesis, Structural Investigation, Thermal Decomposition Mechanism and Sensitivity Properties of an Energetic Compound $[\mathrm{Cd}(\mathrm{DAT}) 6](\mathrm{ClO} 4) 2$ (DAT=1,5-diaminotetrazole), J. Hazard. Mater., 2008, 160, 45-50; f) Zhang J.-G., Li Z.-M., Zang Y., Zhang T.-L., Shu Y.-J., Yang L., Power P.P., Synthesis, Structural Investigation and Thermal Properties of a Novel Manganese Complex $\mathrm{Mn}_{2}(\mathrm{DAT})_{2} \mathrm{Cl}_{4}\left(\mathrm{H}_{2} \mathrm{O}\right)_{4}(\mathrm{DAT}=1,5$-diaminotetrazole), J. Hazard. Mater, 2010, 178, 1094-1099; g) Geisberger G., Klapötke T.M., Stierstorfer J., Copper Bis(1-methyl-5-nitriminotetrazolate): A Promising New Primary Explosive, Eur. J. Inorg. Chem., 2007, 4743-4750.

[4] a) Yang Q., Wei Q., Chen S.-P., Zhang G.-C., Zhou C.-S., Gao S.-L., Solid State Synthesis, Thermodynamics and Catalytic Combustion Effect of a High Energy Nickel(II) Coordination Compound, J. Anal. Appl. Pyrolysis, 2013, 99, 66-70; b) Izsák D., Klapötke T.M., Reuter S., Rösener T., Silver Salt and Derivatives of 5-Azido-1H-1,2,4-triazole-3-carbonitrile, Z. Anorg. Allg. Chem., 2013, 639, 899905; c) Cudzilo S., Nita M., Synthesis and Explosive Properties of Copper(II) Chlorate(VII) Coordination Polymer with 4-Amino-1,2,4-Triazole Bridging Ligand, J. Hazard. Mater., 2010, 177, 146-9; d) Cudziło S., Trzciński W., Nita M., Michalik S., Krompiec S., Kruszyński R., Kusz J., Preparation, Crystal Structure and Explosive Properties of Copper(II) Perchlorate Complex with 4-Amino-1,2,4Triazole and Water, Propellants Explos. Pyrotech., 2011, 36, 151-159; e) Wang S.W., Yang L., Feng J.-L., Wu B.-D., Zhang J.-G., Zhang T.-L., Zhou Z.-N., Synthesis, Crystal Structure, Thermal Decomposition, and Sensitive Properties of Two Novel Energetic Cadmium(II) Complexes Based on 4-Amino-1,2,4-triazole, Z. Anorg. Allg. Chem., 2011, 637, 2215-2222; f) Zhang G.-F., Gao L., Li P., Zhao F.-Q., Fan X.-Z., Chen N., Xiang X.-P., Gao H.-C., Wang J., Structural Diversity in Trinuclear Nickel(II) Complexes of 3,5-Diamino-1,2,4-Triazole, J. Coord. Chem., 2011, 64, 3551-3559.

[5] a) Li S., Wang Y., Qi C., Zhao X., Zhang J., Zhang S., Pang S., 3D Energetic Metal-Organic Frameworks: Synthesis and Properties of High Energy Materials, Angewandte Chemie Int. Ed., 2013, 52, 1-6; b) Zhang Q., Shreeve J.M., Metal- 
Organic Frameworks as High Explosives: A New Concept for Energetic Materials, Angewandte Chemie Int. Ed., 2014, 53, 2540-2542; c) Zhang S., Liu X., Yang Q., Su Z., Gao W., Wei Q., Xie G., Chen S., Gao S., A New Strategy for Storage and Transportation of Sensitive High-Energy Materials: Guest-Dependent Energy and Sensitivity of 3D Metal-Organic-Framework-Based Energetic Compounds, Chem. Eur. J., 2014, 20, 7906-7910.

[6] Gou Y.-T., Yue F., Chen H.-M., Liu G., Sun D.-C., Syntheses and Structures of 3d-4d Heterometallic Coordination polymers Based on 4-Amino-1,2,4-Triazole, J. Coord. Chem., 2013, 66, 1889-1896.

[7] Xu C.-X., Yin X., Jin X., He P., Qin J., Zhang J.-G., Jiao J.-S., Two Coordination Polymers with 3-Hydrazino-4-amino-1,2,4-triazole as Ligand: Synthesis, Crystal Structures, and Non-Isothermal Kinetic Analysis, J. Coord. Chem., 2014, 67, 20042015.

[8] Yang Q., Xie G., Chen S., Gao S., Synthesis, Structure and Thermodynamics of Supermolecular Compound [Ni3(Hdatrz)6(sca)2(H2O)4]sca $11 \mathrm{H} 2 \mathrm{O}$ $($ Hdatrz $=3,5$-diamino-1,2,4-triazole, H2sca $=$ succinic acid), J. Therm. Anal. Calorim., 2011, 110, 979-984.

[9] a) Konovalova I.S., Shishkina S.V., Paponov B.V., Shishkin O.V., Analysis of the Crystal Structure of Two Polymorphic Modifications of 3,4-Diamino-1,2,4Triazole Based on the Energy of the Intermolecular Interactions, Cryst. Eng. Comm., 2010, 12, 909-916; b) Konovalova I.S., Nelyubina Y.V., Lyssenko K.A., Paponov B.V., Shishkin O.V., Intra- and Intermolecular Interactions in the Crystals of 3,4-Diamino-1,2,4-triazole and Its 5-Methyl Derivative. Experimental and Theoretical Investigations of Charge Density Distribution, J. Phys. Chem. A, 2011, 115, 8550-8562; c) Naik A.D., Tinant B., Léonard A., Marchand-Brynaert J., Su B.-L., Garci Y., (Di)-aminoguanidine Functionalization through Transamination: an Avenue to an Auspicious Class of Supramolecular Synthons, Cryst. Growth Des., 2011, 11, 4034-4043.

[10] Jin X., Zhang J.-G., Xu C.-X., Yin X., He P., Qin Q., Eco-friendly Energetic Complexes Based on Transition Metal Nitrates and 3,4-Diamino-1,2,4-triazole (DATr), J. Coord. Chem., 2014, 67, 3202-3215.

[11] Wu J.-T., Zhang J.-G., Yin X., Sun M., Zhang T.-L., Synthesis, Characterization, and Thermal Analysis of Two Energetic Ionic Salts Based on 3,4-Diamino-1,2,4triazole (DATr), Z. Anorg. Allg. Chem., 2013, 639, 2354-2358.

[12] Sheldrick G.M. SHELXS-97, University of Göttingen: Germany: 1990.

[13] Sheldrick G.M. SHELXL-97, University of Göttingen: Germany: 1997.

[14] Frisch M.J., Trucks G.W., Schlegel H.B., Scuseria G.E., Robb M.A., Cheeseman J.R., Montgomery J.A., Jr., Vreven T., Kudin K.N., Burant J.C., Millam J.M., Iyengar S.S., Tomasi J., Barone V., Mennucci B., Cossi M., Scalmani G., Rega N., Petersson G.A., Nakatsuji H., Hada M., Ehara M., Toyota K., Fukuda R., Hasegawa J., Ishida M., Nakajima T., Honda Y., Kitao O., Nakai H., Klene M., Li X., Knox J. E., Hratchian H. P., Cross J. B., Bakken V., Adamo C., Jaramillo J., Gomperts R., Stratmann R.E., Yazyev O., Austin A.J., Cammi R., 
Pomelli C., Ochterski J.W., Ayala P.Y., Morokuma K., Voth G.A., Salvador P., Dannenberg J.J., Zakrzewski V.G., Dapprich S., Daniels A.D., Strain M.C., Farkas O., Malick D.K., Rabuck A.D., Raghavachari K., Foresman J.B., Ortiz J.V., Cui Q., Baboul A.G., Clifford S., Cioslowski J., Stefanov B.B., Liu G., Iiskorz P., Komaromi I., Martin R.L., Fox D.J., Keith T., Al-Laham M.A., Peng C.Y., Nanayakkara A., Challacombe M., Gill P.M.W., Johnson B., Chen W., Wong M.W., Gonzalez C., Pople J.A., GAUSSIAN 9 (Revision A.01): Gaussian, Inc, 2009.

[15] Lu T. Multiwfn. http://multiwfn.codeplex.com/.

[16] Alghool S., Slebodnick C., One Dimensional Structure of Zn(II) Metal Organic Framework (MOF) Assembled Rapidly at Room Temperature: Structural, Thermal Study, and Luminescent Properties, J. Inorg. Organomet. Polym. Mater., 2014, 24 , 644-651.

[17] a) Tao G.-H., Huang Y., Boatz J.A., Shreeve J.M., Energetic Ionic Liquids based on Lanthanide Nitrate Complex Anions, Chem. Eur. J., 2008, 14, 11167-11173; b) Steinhauser G., Klapötke T.M., "Green” Pyrotechnics: A Chemists' Challenge, Angew. Chem., Int. Ed., 2008, 47, 3330-3347.

[18] Kissinger H.E., Anal. Chem., 1957, 29, 1702-1706.

[19] Ozawa T., Bull. Chem. Soc. Jpn., 1965, 38, 1881-1886.

[20] a) Suceska M., Test Methods for Explosives, Springer, New York, 1995; p. 21 (impact), p. 27 (friction); b) Walley S.M., Field J.E., Biers R.A., Proud W.G., Williamson D.M. Jardine A.P., Propell. Explos. Pyrotech. 2015, 40, 351-365.

[21] Karaghiosoff K., Klapötke T.M., Miró Sabaté C., Energetic Silver Salts with 5-Aminotetrazole Ligands, Chem. Eur. J., 2009, 15, 1164-1176.

[22] Fischer D., Klapötke T.M., Piercey D.G., Stierstorfer J., Copper Salts of Halo Tetrazoles: Laser-Ignitable Primary Explosives, J. Energ. Mater., 2012, 30, 40-54.

[23] Impact: insensitive $>40 \mathrm{~J}$, less sensitive $\geq 35 \mathrm{~J}$, sensitive $\geq 4 \mathrm{~J}$, very sensitive $\leq 3 \mathrm{~J}$; Friction: insensitive $>360 \mathrm{~N}$, less sensitive $=360 \mathrm{~N}$, sensitive $<360 \mathrm{~N}$ and $>80 \mathrm{~N}$, very sensitive $\leq 80 \mathrm{~N}$, extremely sensitive $\leq 10 \mathrm{~N}$, According to: Recommendations on the Transport of Dangerous Goods, Manual of Tests and Criteria, $4^{\text {th }}$ ed., United Nations, New York, 1999. 